\title{
Decisional impairments in cocaine addiction, reward bias, and cortical oscillation "unbalance"
}

\author{
This article was published in the following Dove Press journal: \\ Neuropsychiatric Disease and Treatment \\ 20 March 2015 \\ Number of times this article has been viewed
}

\section{Michela Balconi \\ Roberta Finocchiaro}

Research Unit in Affective and Social Neuroscience, Department of Psychology, Catholic University of the Sacred Heart, Milan, Italy
Correspondence: Michela Balconi Catholic University of Milan, Largo Gemelli, I, 20I23, Milan, Italy

Tel +390272342233

$\mathrm{Fax}+390272342769$

Email michela.balconi@unicatt.it
Abstract: A vast amount of research has suggested that subjects with substance use disorder (SUD) might have difficulty making advantageous decisions that opt in favor of a longer-term, larger reward than an immediate, smaller reward. The current research explored the impact of reward bias and cortical frontal asymmetry (left lateralization effect) in SUD in response to a decisional task (Iowa Gambling Task). Fifty SUD participants and 40 controls (CG) were tested using the Iowa Gambling Task. Electrophysiology (electroencephalography) recording was performed during task execution. We measured left and right dorsolateral prefrontal cortex power activity. Behavioral responses (gain/loss options); frequency band modulation (asymmetry index) for delta, theta, alpha, and beta band; and cortical source localization (standardized low-resolution brain electromagnetic tomography) were considered. The SUD group opted in favor of the immediate reward option (loss) more frequently than the long-term option (gain) when compared to the CG. Secondly, SUD showed increased left-hemisphere activation in response to losing (with immediate reward) choices in comparison with the CG. The left hemispheric unbalance effect and the "reward bias" were adduced to explain the decisional impairment in SUD.

Keywords: drug addiction, cortical brain oscillations, left lateralization effect, reward mechanism, Iowa Gambling Task

\section{Introduction}

Recently, some studies have tried to determine whether gambling behavior and drug addiction are related to neurobiological dysfunctions, and whether these dysfunctions are similar to those observed in individuals with prefrontal neurological impairment. ${ }^{1,2}$ Indeed, these studies showed that gambling disorder is associated with deficits in frontal lobe function, and that gambler subjects showed behavior similar to that of subjects who have substance use disorder (SUD): both prefer choices that bring immediate reward, even if these choices are coupled with negative future outcomes. ${ }^{3,4}$ Therefore, compulsive drug use can be described as a condition associated with dysfunctional brain mechanisms that subvert the ability to make decisions. ${ }^{5-7}$ Indeed, high reward sensitivity was shown to contribute to drug abuse vulnerability. ${ }^{8-10}$ A vast amount of research has suggested that those with SUD might have difficulty making advantageous decisions that opt in favor of a longer-term, larger reward than an immediate, smaller reward. ${ }^{9,11,12}$ That is, dysfunctional behavior could arise from poor decision-making skills that lead individuals with SUD to ignore long-term negative consequences in the interest of immediate gratification or relief from uncomfortable states. ${ }^{13}$ Indeed, a tendency was observed for people with SUD: when confronted with a decision that involves a conflict between an immediate reward, which has a long-term and possibly negative consequence (no larger reward later), individuals with SUD chose the 
immediate reward at the expense of negative consequences (or the "delay discounting" effect; for a review on this topic, see Reynolds ${ }^{14}$ ).

However, it is important to identify and clarify the neural substrates that underlie dysfunctional decision making. This may elucidate mechanisms contributing to continued gambling behavior in SUD. Indeed, addictive behavior seems to be associated with specific dysfunctions in the dopaminergicmesolimbic reward system, which can elicit conditioned attention allocation for dependence-associated stimuli, rendering them especially salient. ${ }^{15}$ Deficient mesolimbic reward system and prefrontal cortex (PFC) activation is reported in substance abusers and impulsive individuals. ${ }^{16-18}$ Moreover, it was shown that subjects displayed significantly reward-related and riskier decision making after disruption of the right lateral PFC, choosing a larger potential reward, even at a greater risk of penalty. ${ }^{19,20}$

It should be hypothesized that the hemispheric "unbalance" between the left and right PFC would characterize the subjects' decisions, which shows a higher reward trait and riskier behavior with a possible left-hemisphere unbalance. While the left PFC was shown to be implicated in approach-related and reward-related motivations and emotions, the right PFC was found to be involved in withdrawal-related motivations and emotions. ${ }^{21-24}$ Both approach and withdrawal motivations are paralleled by reward and punishment contingencies.

In this regard, modulation of brain oscillations may be considered a valid measure of brain lateralized activation, often being applied to find distinct responsiveness in the two hemispheres for different cognitive or emotional tasks. ${ }^{21,25}$ Indeed, resting frontal electrophysiology (electroencephalography [EEG]) asymmetry has been hypothesized to relate to appetitive (approach-related) and aversive (withdrawalrelated) motivation and emotion, with heightened approach tendencies reflected in left frontal activity and heightened withdrawal tendencies reflected in relative right frontal activity. ${ }^{26,27}$ In the frontal system, reduction in a specific frequency band - that is, a reduction of alpha power (increased activity) in the left frontal brain - was found after money gain and reward trials, whereas punishment conditions induced a reduction in alpha power in the right frontal brain. ${ }^{28-30}$ Previous research on resting EEG has shown that frontal hemispheric activation asymmetry in favor of the left PFC reflects an individual predisposition to respond in terms of withdrawal-related behavior. ${ }^{23,24}$ One previous study used the resting alpha level to analyze the effect of stable approach/ withdrawal attitudes on decisional behavior. ${ }^{31}$ Another study used a wider band of frequencies. ${ }^{32}$ This latter case also found that baseline cortical activity in the right hemisphere predicts individual risk-taking behavior. The subjects with higher baseline cortical activity in this area display more risk aversion than do other subjects. It was also suggested that hypoactivity in the right PFC might serve as a dispositional marker of greater risk-taking behavior. Therefore, it should be plausible that the hemispheric "unbalance" between the left and right sides would characterize the subjects' risktaking behavior, showing a higher reward attitude with an unbalance in favor of the left hemisphere.

\section{Purpose}

In the present work, we tried to explore the lateralization effect - that is, the contribution by the left hemisphere to the reward-related components that support dysfunctional behavior in SUD. The role of the frontal brain unbalance was supposed to be able to elucidate the deficitary decisional mechanisms in SUD. As shown by previous research, left reward-related brain asymmetry (alpha band modulation) was observed in SUD in response to more rewarding conditions. ${ }^{28}$ Some studies found a significant relationship between theta and gamma oscillations and the reward system. ${ }^{33}$ However, a specific relationship with dysfunctional decisional processes was not explored, taking into account dynamic patterns (not resting EEG) and an ample range of brain oscillations (in addition to alpha band) during decisional task execution. In addition, the exploration of an ample range of brain oscillations allows for a complete analysis of the functional significance of potential brain modulation in relationship with specific cortical sites where this modulation is observed.

Taken together, these features may predict a significant sensitivity toward more rewarding options for SUD, and a final strategy that is more oriented toward immediate rewards and risky choices. In the present research, we intend to analyze this sort of hypersensitivity to reward and insensitivity to punishment, which should be related to the inability to attribute an adequate relevance to the loss (reward violation) in the behavioral strategy used. Secondly, we may suppose that, based on the lateralized reward/punishment model, there are different contributions by the left and right hemispheres on decisional processes, including gains and losses. Thus, that SUD increased responsiveness to more immediate "rewarding" choices may be supported by the unbalance between the left and the right hemispheres, favoring the left hemisphere. We proposed that, in association with riskier choices, SUD subjects showed an increase in high-frequency band power (brain activity increases) versus low-frequency band power (brain activity decreases) within the left hemisphere in comparison 
with normal subjects. Specifically, we supposed that SUD subjects showed an increased power of the high-frequency band (beta) within the left hemisphere in case of an immediate reward (disadvantageous decks [DD]) in comparison with a delayed reward (advantageous decks [AD]) because this increased power should represent higher left hemispheric activity. On the contrary, low-frequency bands delta, theta, and alpha should be decreased within the left hemisphere in cases of DD in comparison with AD because the decrease of the low-frequency oscillations should support the increased responsiveness of the left side. Finally, a source localization analysis (standardized low-resolution brain electromagnetic tomography [sLORETA]) was applied to the EEG data to confirm cortical brain activity localization.

\section{Methods}

\section{Subjects}

The SUD group included 50 individuals (see Table 1 for sociodemographic data). These patients were selected from the Drug Dependence Department, SERT 11, Monza, where they were treated for their dependent behavior. They met the criteria for a lifetime history of cocaine dependence based on responses on the Structured Clinical Interview for Diagnostic and Statistical Manual of Mental Disorders (DSM)-IV (SCID-II). ${ }^{34}$ Before any testing, drug users were interviewed to collect information about their drug of choice ("which drug has caused more problems to you in the past?"). The interview was also used to examine the homogeneity for severity of drug use and its time duration. The interview evaluates three parameters: the intensity (average dosing); frequency (consumption episodes by month); and duration (years) of the use of a series of substances (alcohol, amphetamines, cannabis, cocaine, heroin, and methadone). Based on these criteria, all the selected subjects were cocaine dependent with a severe profile (see Table 1). Urine analyses for cannabis, benzodiazepines, cocaine, and heroin metabolites were routinely conducted in order to confirm the abstinence period.

The control group (CG) included 40 individuals who were recruited using a previous dataset of voluntary subjects; they

Table I Demographic features by group

\begin{tabular}{lll}
\hline & SUD & CG \\
\hline Age, mean (SD) & $53.3(4.33)$ & $52.10(3.89)$ \\
Education, mean (SD) & $12.34(2.73)$ & $12.11(2.34)$ \\
Sex (\% female), mean (SD) & $53.32(2.98)$ & $54.32(2.75)$ \\
Race & White & \\
Years of regular substance & $14.42(2.54)$ & \\
abuse, mean (SD) & & \\
\hline
\end{tabular}

Abbreviations: SUD, substance use disorder; CG, control group. had no history of use of illicit drugs, no lifetime regular use of alcohol, no more than 3 days of alcohol use in the last 30 days, and no more than two drinks per drinking day. In addition, CG was matched with the SUD group with respect to age, race, sex, and education.

Exclusion criteria for both SUD and CG included suicidal ideation and psychosis. Also, the absence of documented comorbid mood or personality disorders (for depression, the Beck Depression Inventory [BDI-II] by Beck et $\mathrm{al}^{35}$ was used), the absence of documented head injury or neurological disorders, and a minimum abstinence duration of 20 days before testing were considered exclusion criteria. All subjects were right-handed. The subjects gave informed written consent for participating in the study and the research was approved by the ethics committee at the institution where the work was carried out (Department of Psychology, Ethics Committee of the Catholic University of Milan, Milan, Italy). The study conforms to the code of ethics of the World Medical Association (Declaration of Helsinki) printed in the British Medical Journal. No incentives were given to the subjects (both SUD and controls) to participate in the research.

\section{Procedure}

The Iowa Gambling Task (IGT) (adaptation for an online Italian version) was used. ${ }^{36-38}$ The IGT is a sensitive measure of decision making that simulates a real-world decision-making situation requiring evaluation of the magnitude and timing of rewards and punishments under uncertain conditions. The IGT includes a number of aspects: immediate rewards and delayed punishments; risk; and uncertainty of outcomes. Subjects were instructed to try to gain as much money as possible by drawing 100 trials from a choice of four decks (starting with a fictive loan of $€ 2,000$ ). Two of the decks were more risky and disadvantageous (A and B), producing immediate large rewards, but they were accompanied by significant money loss due to extreme punishments. The remaining two decks were advantageous (C and D) because the rewards were modest, but more consistent and the punishment was low. Therefore, high-risk options implied that there was the chance of a great reward, but there was also high risk for loss (DD). By contrast, low-risk options are often characterized by lower reward, but also low risk for loss (AD). Thus, the low-risk options often entail better long-term strategies with an overall gain, despite the initial reduced short-term gain.

All participants received standard instructions for the IGT. They were told that the object of the task was to win as much as possible and to avoid losses by drawing cards, one at a time, from the four decks. They were informed that 
each card drawn would indicate how much they had won and whether there was a penalty as well. They were also informed that some decks were more advantageous than others, and that they were free to switch from one deck to another at any time and as frequently as they liked. They were not informed of the schedule of wins and losses in each deck or given advice on how to draw the cards, nor were they told how many cards they would be allowed to draw.

Each subject sat on a comfortable chair in front of a computer screen $(1,280 \times 1,024$ pixels $)$. The screen was placed approximately $80 \mathrm{~cm}$ from the subject, with a visual horizontal angle of $4^{\circ}$ and a vertical angle of $6^{\circ}$. They used a Stimpad to choose the card with no strict time restrictions, although they were required to decide within a brief time interval. The experimental session lasted about 1.5 hours. During the task, the EEG was recorded.

\section{Behavioral measure}

The behavioral measure included the 100 card selections. We counted the number of selections from decks A and B (disadvantageous) and the number of selections from decks $\mathrm{C}$ and D (advantageous) and then derived the net score,

$$
[(\mathrm{C}+\mathrm{D})-(\mathrm{A}+\mathrm{B})]
$$

(response index [rI]). ${ }^{4}$ We subdivided the 100 card selections into five blocks of 20 cards each, and for each block, a specific rI was calculated.

\section{EEG recordings and data reduction}

EEG recordings were performed with a 64-channel directcoupled amplifier (SynAmps system) and acquisition software (Neuroscan 4.2) during task execution. An ElectroCap with $\mathrm{Ag} / \mathrm{AgCl}$ electrodes was used to record EEGs from active scalp sites referred to the earlobes (10/20 system of electrode placement). ${ }^{39}$ Data were acquired using a sampling rate of $500 \mathrm{~Hz}$, with a frequency band of 0.01-50 Hz. An offline common average reference was successively computed to limit the problems associated with the signal-to-noise ratio. ${ }^{40}$ Additionally, two electrooculography electrodes were placed on the outer canthi to detect eye movements. The impedance of the recording electrodes was monitored for each subject prior to data collection and was always below $5 \mathrm{k} \Omega$. The signal was visually scored, and a portion of the data that contained artifacts were removed to increase specificity. Blinks were also visually monitored. Ocular artifacts (eye movements and blinks) were corrected using an eye-movement correction algorithm that employs a regression analysis in combination with artifact averaging. ${ }^{41}$ After performing electrooculography correction and visual inspection, only artifact-free trials were considered (rejected epochs, $2 \%$ ).

We measured left and right frontal dorsolateral (DL) PFC (F3, F4) power activity. The digital EEG data (from all 64 active channels) were band-pass filtered in the following frequency bands: delta $(0.5-4 \mathrm{~Hz})$, theta $(4-8 \mathrm{~Hz})$, alpha (8-12 Hz), and beta (14-20 Hz) (band-pass filtering: $96 \mathrm{~dB} /$ octave roll-off; warm-up filter left and right to $100 \mathrm{~ms}$ ). To obtain a signal proportional to the power of the EEG frequency band, the filtered signal samples were squared. An average absolute power value for each condition (AD and DD) was calculated, using the time window of 0-500 ms. A fast Fourier transform method (Hamming window: length $10 \%)$ was used to obtain estimates of spectral power $\left(\mu \mathrm{V}^{2}\right)$ in the $1 \mathrm{~Hz}$ frequency bin for each electrode site. Spectral power values were averaged across all epochs within a single baseline and were then transformed to power density values for the different frequency bands. All power density values were log-transformed to normalize the distribution of the data after the subtraction.

\section{Results}

The statistical analysis included three different sets: a first analysis applied to the behavioral data (rI) to explore the significant differences during the IGT; a second analysis related to the frequency band differences for SUD and CG; and a third set related to the frequency band distribution on the scalp based on the sLORETA analysis.

\section{$\mathrm{rl}$}

The behavioral measures were subjected to a two-way analysis of variance (ANOVA), in which the between-subjects factor group (2; SUD versus CG) and the within-subjects factor block (5) were applied to the rI. Errors associated with inhomogeneity of variance were controlled by decreasing the degrees of freedom using the Greenhouse-Geiser epsilon (Table 2). A significant main effect was found for group $\left(F[1,88]=13.56 ; P=0.001 ; \eta^{2}=0.40\right)$, block $(F[4,88]=15.23$; $\left.P=0.001 ; \eta^{2}=0.43\right)$, and group $\times$ block $(F[4,88]=16.57$; $P=0.001 ; \eta^{2}=0.44$ ) (Figure 1 ). As revealed by post hoc analysis (contrast analysis for ANOVA, with Bonferroni corrections for multiple comparisons), a higher rI was found for the CG compared to those with SUD within all five blocks (all $P<0.001)$. Moreover, the $\mathrm{CG}$ showed significant differences when comparing the five intervals, with an increased performance observed across the intervals (all $P<0.001$ ), whereas the SUD group did not show any significant effect. 
Table 2 Mean and SD values of $\mathrm{rl}$ as a function of group (SUD versus $(G)$ and block

\begin{tabular}{lll}
\hline & \multicolumn{1}{rl}{} & \\
\cline { 2 - 3 } & Mean & SD \\
\hline SUD & -0.05 & \\
Block I & -0.11 & 0.02 \\
Block 2 & -0.03 & 0.02 \\
Block 3 & 0.06 & 0.01 \\
Block 4 & 0.05 & 0.03 \\
Block 5 & & 0.04 \\
CG & -0.08 & \\
Block 1 & 1.89 & 0.03 \\
Block 2 & 3.17 & 0.05 \\
Block 3 & 5.12 & 0.02 \\
Block 4 & 6.90 & 0.08 \\
Block 5 & & 0.05 \\
\hline Abbrevitions SD, stin
\end{tabular}

Abbreviations: SD, standard deviation; rl, response index; SUD, substance use disorder; CG, control group.

\section{Frequency band analysis (delta, theta, alpha, beta)}

The frontal brain log-transformed asymmetry (LTA)

[log-transformed right power - log-transformed left power]

for mean power was calculated. A negative value indicated a more left-sided increase in power for the specific frequency band. Conversely, a more positive value indicated a more left-sided decrease in power for the frequency band.

Each frequency band was subjected to a two-way mixeddesign ANOVA, in which the between-subjects group (2; SUD versus CG) and within-subjects condition (2; AD versus DD) were applied to the dependent variable of the LTA (Table 3).

For delta, a significant group $\times$ condition interaction effect was found $\left(F[1,88]=10.04 ; P=0.001 ; \eta^{2}=0.40\right)$ (Figure 1A). Specifically, as shown by post hoc comparisons (contrast analysis for ANOVA, with Bonferroni corrections for multiple comparisons), an increased LTA (positive values, more delta decreasing within the left hemisphere) was found for the SUD group than the CG in response to DD $(F[1,88]=9.70$; $\left.P=0.001 ; \eta^{2}=0.38\right)$.

For theta, a significant group $\times$ condition interaction effect was found $\left(F[1,88]=8.04 ; P=0.001 ; \eta^{2}=0.37\right)$ (Figure 1B). As revealed by contrast analysis, an increased LTA (positive values; more theta decreasing within the left hemisphere) was found for the SUD group when compared to the CG in case of $\mathrm{DD}\left(F[1,88]=9.13 ; P=0.001 ; \eta^{2}=0.39\right)$.

For alpha, a significant group $\times$ condition interaction effect was found $\left(F[1,88]=11.16 ; P=0.001 ; \eta^{2}=0.41\right)$ (Figure 1C). Indeed, there was a significantly increased LTA
A

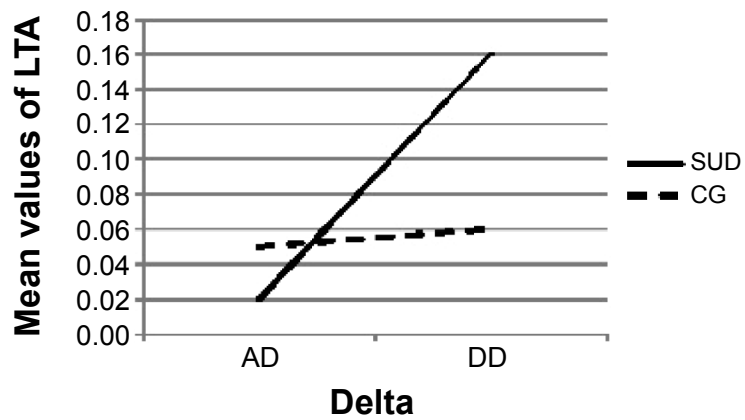

B

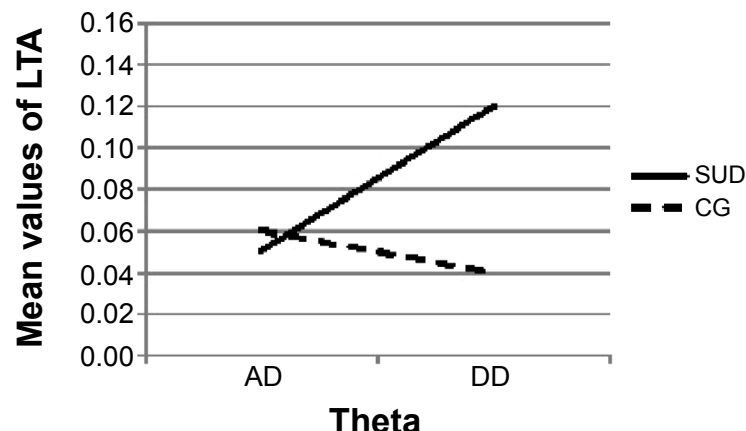

C

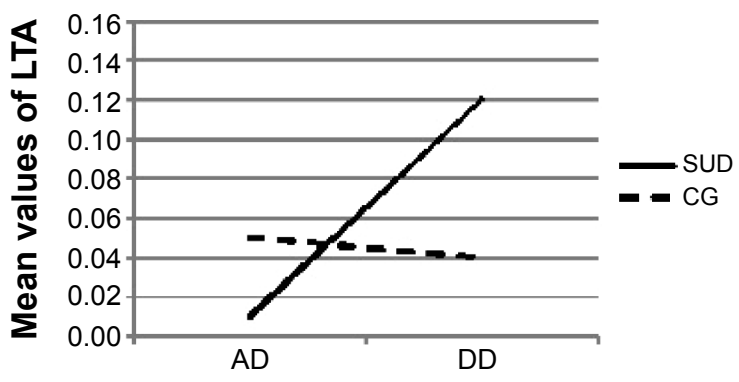

Alpha

D

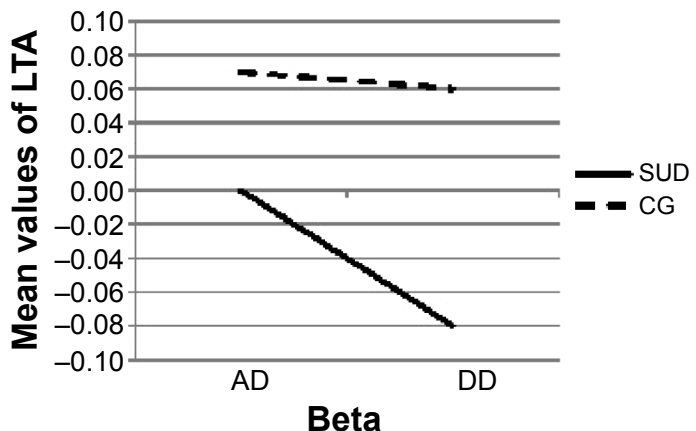

Figure I Mean values of LTA for each frequency band distinctly for the SUD group and CG as a function of condition (AD versus DD).

Notes: (A) Delta; (B) theta; (C) alpha; and (D) beta.

Abbreviations: AD, advantageous decks; DD, disadvantageous decks; SUD, substance use disorder; CG, control group; LTA, log-transformed asymmetry. 
Table 3 Mean and SD values of LTA as a function of group (SUD versus CG) and condition (AD versus DD) for each frequency band

LTA

\begin{tabular}{|c|c|c|c|c|c|c|c|c|}
\hline \multirow[t]{3}{*}{ Frequency band } & \multicolumn{4}{|l|}{ SUD } & \multicolumn{4}{|l|}{ CG } \\
\hline & \multicolumn{2}{|l|}{ AD } & \multicolumn{2}{|l|}{ DD } & \multicolumn{2}{|l|}{ AD } & \multicolumn{2}{|l|}{ DD } \\
\hline & Mean & SD & Mean & SD & Mean & SD & Mean & SD \\
\hline Delta & 0.02 & 0.003 & 0.16 & 0.001 & 0.05 & 0.002 & 0.06 & 0.001 \\
\hline Theta & 0.05 & 0.002 & 0.13 & 0.004 & 0.06 & 0.002 & 0.04 & 0.002 \\
\hline Alpha & 0.01 & 0.002 & 0.12 & 0.003 & 0.05 & 0.001 & 0.04 & 0.003 \\
\hline Beta & 0.03 & 0.001 & -0.08 & 0.004 & 0.07 & 0.002 & 0.06 & 0.001 \\
\hline
\end{tabular}

Abbreviations: SD, standard deviation; LTA, log-transformed asymmetry; SUD, substance use disorder; CG, control group; AD, advantageous decks; DD, disadvantageous decks.

(positive values; more alpha decreasing within the left hemisphere) for the SUD group compared to the $\mathrm{CG}$ in response to $\mathrm{DD}\left(F[1,88]=10.08 ; P=0.001 ; \eta^{2}=0.40\right)$.

For beta, the results showed a significant group $\times$ condition interaction effect $\left(F[1,88]=13.33 ; P=0.001 ; \eta^{2}=0.46\right)$ (Figure 1D). As shown by contrast analyses, there was an increase in the LTA (negative values; more beta increasing within the left hemisphere than the $\mathrm{CG}$ in response to DD than AD) for the SUD group $(F[1,88]=9.31 ; P=0.001$; $\left.\eta^{2}=0.40\right)$.

\section{sLORETA analysis}

To localize the source of neural activity, we used the sLORETA method. ${ }^{42,43}$ This method computes the current density (area $/ \mathrm{m}^{2}$ ) according to the digitized probability atlas as the linear, weighted sum of the scalp electric potentials, and it assumes neither a limited number of dipolar point sources, nor distribution on a known surface. It solves the inverse problem based on the assumption that the smoothest possible activity distribution is the most plausible one. Topographical voltage maps of bands were made by plotting color-coded isopotentials obtained by interpolating voltage values between scalp electrodes at specific time intervals (0-500 ms). In the present study, an improved version of the standardized weighted sLORETA was applied. ${ }^{44}$ The source space used five-point grid spacing, and the estimated signalto-noise ratio was 3 . In the present research, we calculated the source localization for every subject and condition. Voxel-wise nonparametric statistics were used. Direct comparisons were successively conducted between the groups (SUD versus CG), and distinctively for each condition (DD versus $\mathrm{AD}$ ) and for each frequency band.

For delta and theta, the algorithm localized the source of the differential activation in the left DLPFC for DD between the SUD group and the CG $(t=9.03, P \leq 0.01$, BA9 $\mathrm{x}=-3, \mathrm{y}=48, \mathrm{z}=18 ; t=7.90, P \leq 0.01, \mathrm{BA} 9 \mathrm{x}=-5, \mathrm{y}=45, \mathrm{z}=23)$ (Figure 2A-D). For alpha, more significant differential activation was found in the DLPFC when comparing the SUD group and the $\mathrm{CG}$ for $\mathrm{DD}(t=5.10, P \leq 0.01, \mathrm{BA} 9 \mathrm{x}=2, \mathrm{y}=40, \mathrm{z}=27)$. Finally, beta showed the source of the differential activation within the left DLPFC $(t=5.69, P \leq 0.01$, BA9 $\mathrm{x}=-7, \mathrm{y}=39$, $\mathrm{Z}=30$ ) when comparing the SUD group and the $\mathrm{CG}$ in response to DD. No other effect was statistically significant.

\section{Discussion}

The present study aimed to explore the role of frontal brain asymmetry in decisional choices for both reward and punishment conditions, as well as for drug dependence. Specifically, we considered behavioral (IGT), brain oscillation, and lateralization effects to be related to drug dependence. Frequency band modulation (asymmetry index, LTA) was used to test the left hemispheric unbalance effect in SUD subjects, showing a consistent advantage for the left hemisphere in determining dysfunctional choices. A large range of brain oscillations (delta, theta, alpha, and beta) was considered when exploring the band modulation as a function of the advantageous or disadvantageous behavioral options. Finally, we tested the left hemispheric unbalance in SUD - that is, when SUD subjects opted for the disadvantageous conditions when compared with those in the CG.

Firstly, the results were broadly consistent with the hypotheses that SUD is related to significant deficits in decisional processes based on reward bias. Indeed, SUD and $\mathrm{CG}$ showed different behavioral options and opposite strategies during the IGT. SUD subjects in general increased DD choices in comparison to controls with a constant "gap" between gains and losses across the five blocks. This behavior may be explained by taking into account a possible rewardsensitivity bias. The fact that the SUD group consistently "increased" the response to immediately rewarding events 
A
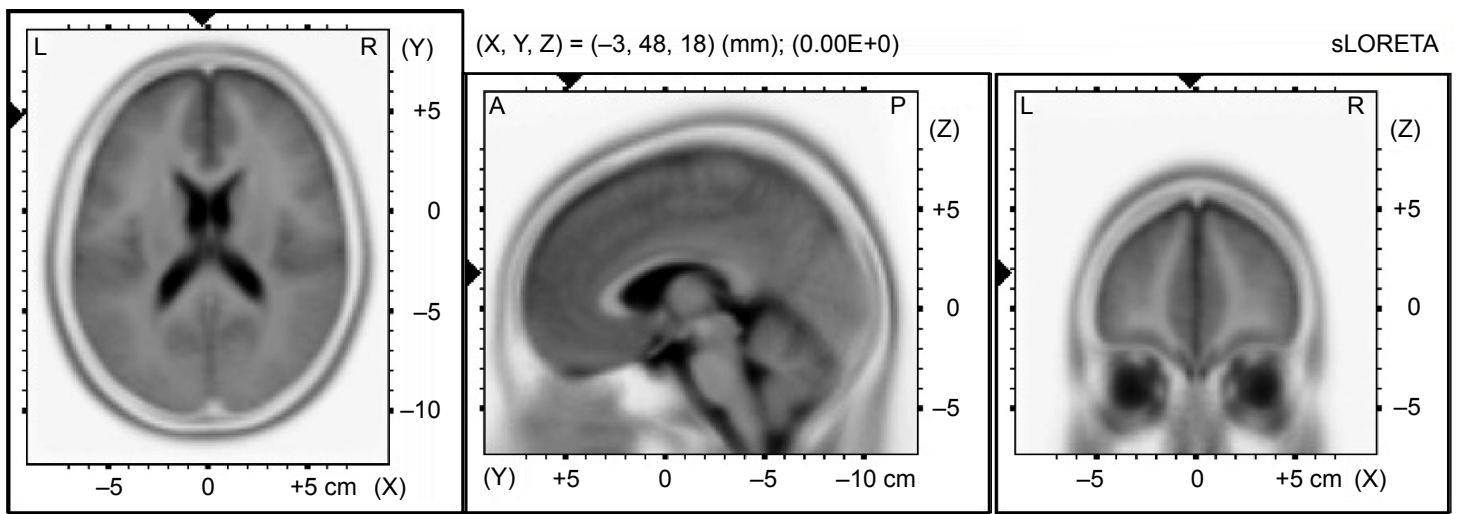

B
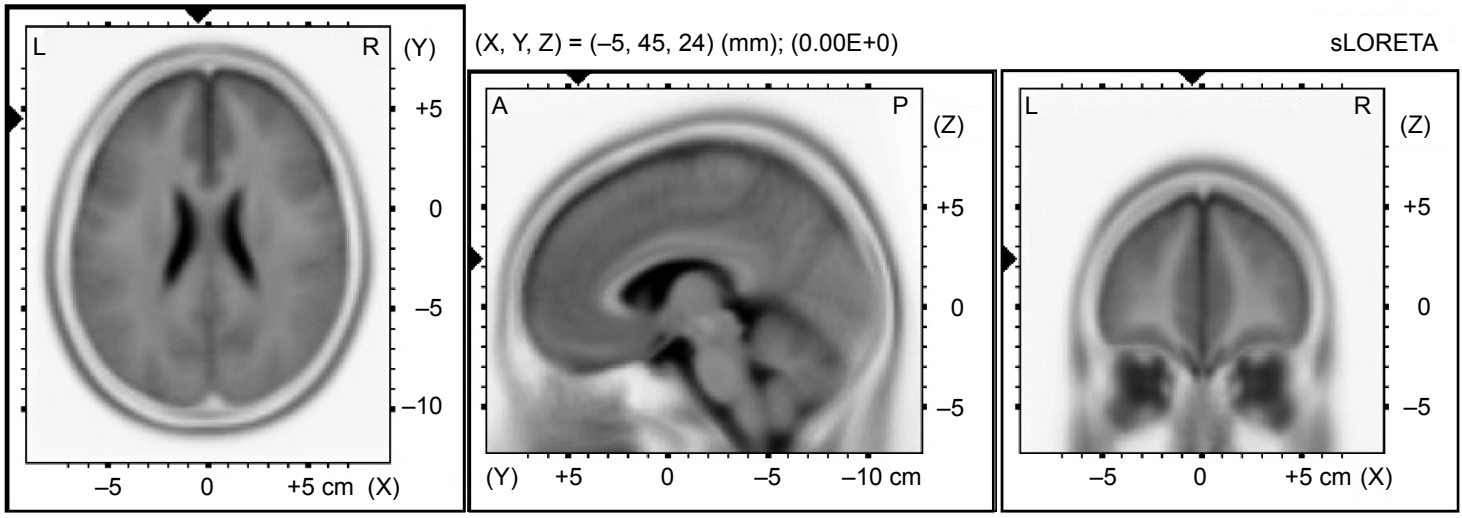

C
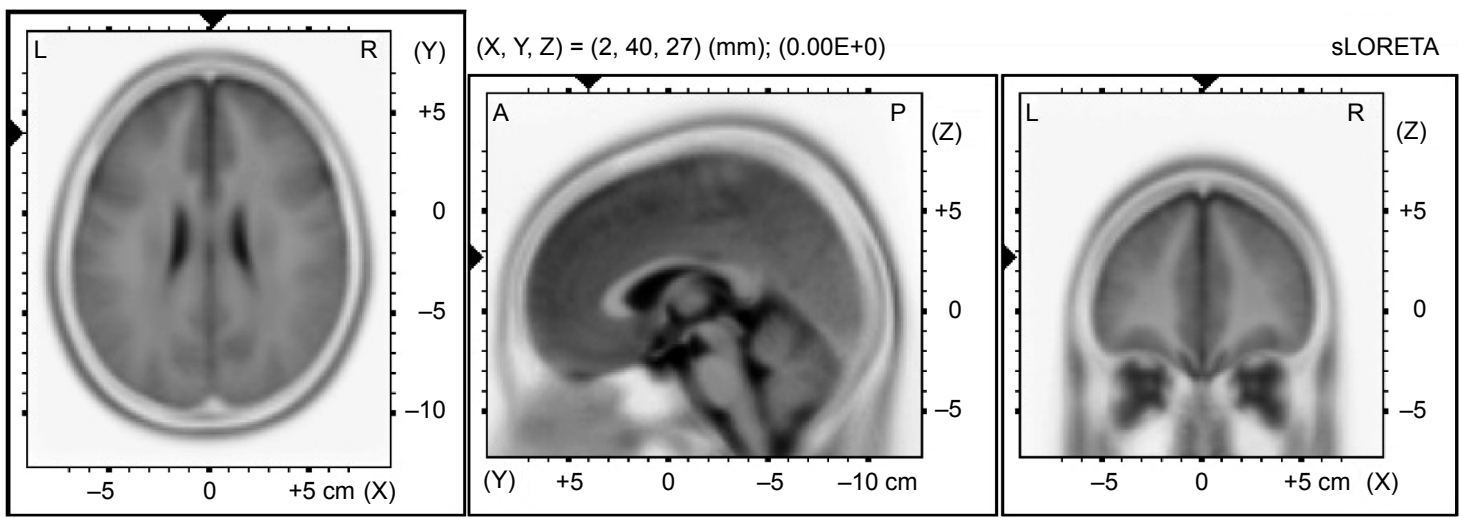

D
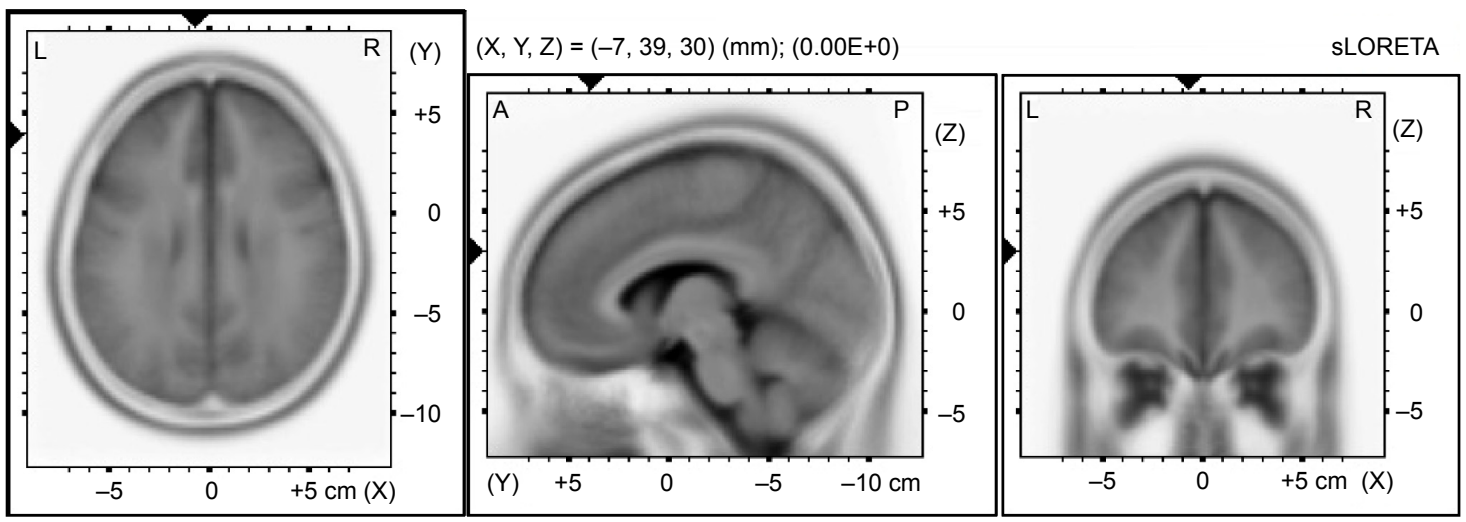

Figure 2 Results of the sLORETA analysis.

Notes: The image shows the sLORETA slices in the Talairach space for the estimated source of activation differences between CG and SUD for DD, respectively, for (A) delta, (B) theta, (C) alpha, and (D) beta.

Abbreviations: sLORETA, standardized low-resolution brain electromagnetic tomography; CG, control group; SUD, substance use disorder; DD, disadvantageous decks. 
as compared to the $\mathrm{CG}$ reinforces the assumption that some motivational impairment is observable in the case of drug dependence. A sort of "reward bias" should be effective when inducing the subject to overestimate the immediate (unreal) reward at a detriment to the delayed (real) reward (delay discounting). ${ }^{13}$ More generally, the reward bias seems to be the most relevant variable able to explain the current results, by pointing out the effect of insensitivity to losses in favor of a more immediate reward. A long-term strategy is inhibited by the apparent present reward outcomes.

A second interesting result of the present study focused on the modulation of the brain oscillations during the IGT execution to monitor the direct effect of the brain lateralization during the dynamic decisional process. Based on the ample range of cortical oscillations we used, we may exhaustively describe the functional significance of these possible modulations as they related to the specific sites where these modulations were revealed. Regarding the hemispheric contribution in affecting the decisional processes, a significant higher left frontal activation for SUD was observed when they opted for DD than AD. The significant decrease in delta, theta, and alpha power within the left hemisphere in response to DD may support the higher left activation because the low-frequency bands were reduced within this side. The delta increasing within the left hemisphere in response to more rewarding conditions confirmed the left-hemisphere overactivation for SUD. The present results may confirm that this increased left response was exclusively related to the DD (it was apparently more rewarding) and it was not generically associated to a resting anomalous left hyperactivation in SUD. Therefore, based on the dynamic index we used (related to the specific deck condition), we may suppose that this left hemispheric unbalance is related to the "rewarding option", induced by the apparent immediate reward. To reinforce these results, it must be noted that the left (and right) brain activity of SUD was equivalent to the CG in cases of nonrewarding (unrisky) options.

Indeed, source analysis (sLORETA) supported these effects, showing a significant increase in the left frontal distribution of this reward-related brain activation for SUD, with decreased slow-frequency (delta and theta) and increased high-frequency (beta) brain oscillations within the frontal left hemisphere. Specifically, we found that the left DLPFC increased in response to more rewarding and risky choices. Thus, we may state that this cortical area could contribute to determine this "impaired" responsiveness to decisional choice in the case of risky options for SUD.
The lateralization effect in SUD related to the brain unbalance in response to specific rewarding conditions may explain why SUD subjects opted for an immediate reward over a future reward (reward bias). This fact affects their sensitivity to contextual cues and their dysfunctional strategic planning. ${ }^{45}$ We have to take into consideration that this left-sided hyperactivation in SUD was related to the case of apparently rewarding choices - that is, more risky and immediately rewarding options (DD). Thus, this fact may support an effective hemispheric unbalance related to increased left sensitivity in response to immediately rewarding (and risky) events, more so than a decreased right responsivity to the delayed rewarding (and unrisky) events.

The specific cortical localization we found may suggest the consistent overactivation of the left cortical system and a concomitant predominance of this brain area in managing the cognitive choices in SUD when they believed they obtained an immediate reward. ${ }^{28}$ Previous research found that the brain correlates of the reward system are mainly localized in prefrontal areas and are more left-lateralized. More generally, the cortical correlates of the reward-inhibition system is the PFC and, while the left PFC was shown to be implicated in approach-related motivations and emotions, the right PFC was found to be involved in withdrawal-related motivations and emotions. ${ }^{21-24}$ Thus, the specific localization that was found may suggest the consistent overimplication of the left cortical system, and a concomitant predominance of this brain area, at least when managing the cognitive choices in SUD. However, these results are partially in contrast with some previous findings about hypoactivity in the right PFC, supporting a sort of neglect for the negative consequences of SUD behavior, ${ }^{46}$ or the idea that a right hypoactivity induces subjects to seek out risks as a means to drive a relatively underactivated system. ${ }^{32}$ Anyway, it should be noted that in a previous study, the EEG resting activity was considered a predictor of the successively more risky or unrisky strategy. ${ }^{6}$ In contrast, the current study focused on the modulation of brain oscillations during the IGT execution to monitor the direct effect of the brain lateralization during the dynamic decisional process. Nevertheless, we cannot exclude a possible mixed effect including both the increased responsiveness in the left hemisphere toward the more rewarding condition, and the decreased responsiveness in the right hemisphere to a less rewarding (or more punishing) condition. Future research may better elucidate this relevant point.

To summarize, the reward bias seems to be the most relevant variable to explain the current results by pointing out the effect of insensitivity to losses in favor of a more 
immediate reward: the long-term strategy is inhibited by the apparent present reward outcomes. The second variable that was found to be critical in explaining the decisional behavior in SUD was the frontal left lateralization of the brain activity in response to selecting more risky options. A sort of higher left hemisphere reactivity by the SUD subjects was revealed, and this possible general interhemispheric unbalance may determine the cognitive bias toward more appetitive and rewarding conditions - that is, this frontal mechanism could have a relevant and consistent effect on the final behavioral strategy based on a biased reward system, producing this dysfunctional general strategy. These results may also support a general conclusion that the most rewarding conditions implicate higher left activation, orienting subjects' choices toward an immediate reward..$^{45,47}$ This frontal cortical network might be able to characterize the subjective behavior in response to decisional processes, when a gain/loss decision must be made. Specifically, the frontal contribution makes relevant the reward system's role in managing the decisional processes. ${ }^{22,31,48,49}$

The "hemisphere unbalance effect" may be considered a critical marker of dysfunctional decisional behavior in drug dependence, and a more general factor that is able to explain the tendency to opt in favor of more reward-related conditions. This frontal mechanism may have a consistent effect on the final behavioral strategy based on a biased reward system, producing dysfunctional general outcomes. Indeed, the partial impairment or the unbalanced contribution of these two sides may compromise the adequacy of future-planning behavior, as has been observed for some specific clinical conditions, such as dependence disorders, ventromedial lesions, or pathological neuropsychiatric categories (including obsessivecompulsive or bipolar personality disorder). ${ }^{50}$

However, based on the present data, the pre-existing stable effect of reward trait (without any modification induced by drug dependence) on risky behavior could also be suggested, although the increasing effect of drug abuse on this reward sensitivity cannot be excluded a priori. In other words, the reward sensitivity shown in SUD could be a sort of facilitating factor that strongly predicts the successive drug abuse and dysfunctional decisional behavior. Future research could better support this supposition.

Moreover, a deep exploration of the potential relationship between the stable left hemispheric hyperactivation in SUD, independently of the context (the more or less rewarding condition), might suggest a more direct relationship between some personality trait component (such as impulsivity) and hemispheric (left) unbalance. In fact, a previous study found that impulsivity was generally related to SUD, and that this effect may induce a higher left dominance. ${ }^{9}$ However, since we did not directly consider the impulsivity construct, and given that our SUD subjects did not show a significant general (baseline) left unbalance, but only a significant more left-lateralized response to more rewarding conditions, the impulsivity component should be better explored (and compared) across groups as a relevant cofactor to verify its effective role.

Finally, our results may also suggest a general limitation in some executive functions in SUD, which is mainly related to the inability to adequately shift the personal cognitive strategy, taking into account the previous outcomes of behavior, as well as to the difficulty in updating the internal and external representations. Future research may point out this aspect to better explain the role of reward bias in relationship with executive dysfunctions.

\section{Disclosure}

The authors report no conflicts of interest in this work.

\section{References}

1. Kalechstein AD, Fong T, Rosenthal RJ, Davis A, Vanyo H, Newton TF Pathological gamblers demonstrate frontal lobe impairment consistent with that of methamphetamine-dependent individuals. J Neuropsychiatry Clin Neurosci. 2007;19(3):298-303.

2. Makris N, Gasic GP, Seidman LJ, et al. Decreased absolute amygdala volume in cocaine addicts. Neuron. 2004;44(4):729-740.

3. Verdejo-García A, Bechara A. A somatic marker theory of addiction. Neuropharmacology. 2009;56 Suppl 1:48-62.

4. Bechara A, Damasio H. Decision-making and addiction (part I): impaired activation of somatic states in substance dependent individuals when pondering decisions with negative future consequences. Neuropsychologia. 2002;40(10):1675-1689.

5. Allen TJ, Moeller FG, Rhoades HM, Cherek DR. Impulsivity and history of drug dependence. Drug Alcohol Depend. 1998;50(2): 137-145.

6. Barry D, Petry NM. Predictors of decision-making on the Iowa Gambling Task: independent effects of lifetime history on substance use disorders and performance on the Trail Making Test. Brain Cogn. 2008;66(3):243-252.

7. Mitchell JM, Fields HL, D'Esposito M, Boettiger CA. Impulsive responding in alcoholics. Alcohol Clin Exp Res. 2005;29(12):2158-2169.

8. Baler RD, Volkow ND. Drug addiction: the neurobiology of disrupted self-control. Trends Mol Med. 2006;12(12):559-566.

9. Bechara A. Decision making, impulse control and loss of willpower to resist drugs: a neurocognitive perspective. Nat Neurosci. 2005; 8(11):1458-1463.

10. Dawe S, Loxton NJ. The role of impulsivity in the development of substance use and eating disorders. Neurosci Biobehav Rev. 2004; 28(3):343-351.

11. Bolla KI, Eldreth DA, London ED, et al. Orbitofrontal cortex dysfunction in abstinent cocaine abusers performing a decision-making task. Neuroimage. 2003;19(3):1085-1094.

12. Verdejo-García A, Pérez-García M. Substance abusers' self-awareness of the neurobehavioral consequences of addiction. Psychiatry Res. 2008;158(2):172-180.

13. Bickel WK, Marsch LA. Toward a behavioral economic understanding of drug dependence: delay discounting processes. Addiction. 2001;96(1): 73-86. 
14. Reynolds B. A review of delay-discounting research with humans: relations to drug use and gambling. Behav Pharmacol. 2006;17(8):651-667.

15. Adinoff B. Neurobiologic processes in drug reward and addiction. Harv Rev Psychiatry. 2004;12(6):305-320.

16. Knyazev GG. Antero-posterior EEG spectral power gradient as a correlate of extraversion and behavioral inhibition. Open Neuroimag $J$. 2010;4:114-120.

17. Limbrick-Oldfield EH, van Holst RJ, Clark L. Fronto-striatal dysregulation in drug addiction and pathological gambling: Consistent inconsistencies? Neuroimage Clin. 2013;2:385-393.

18. Scheres A, Milham MP, Knutson B, Castellanos FX. Ventral striatal hyporesponsiveness during reward anticipation in attention-deficit/ hyperactivity disorder. Biol Psychiatry. 2007;61(5):720-724.

19. Balconi M, Finocchiaro R, Canavesio Y. Left hemispheric imbalance and reward mechanisms affect gambling behavior: the contribution of the metacognition and cortical brain oscillations. Clin EEG Neurosci. Epub 2014 Mar 27.

20. Knoch D, Schneider F, Schunk D, Hohmann M, Fehr E. Disrupting the prefrontal cortex diminishes the human ability to build a good reputation. Proc Natl Acad Sci U S A. 2009;106(49):20895-20899.

21. Balconi M, Mazza G. Brain oscillations and BIS/BAS (behavioral inhibition/activation system) effects on processing masked emotional cues. ERS/ERD and coherence measures of alpha band. Int $J$ Psychophysiol. 2009;74(2):158-165.

22. Balconi M, Mazza G. Lateralisation effect in comprehension of emotional facial expression: a comparison between EEG alpha band power and behavioural inhibition (BIS) and activation (BAS) systems. Laterality. 2010;15(3):361-384.

23. Davidson RJ. What does the prefrontal cortex "do" in affect: perspectives on frontal EEG asymmetry research. Biol Psychol. 2004; 67(1-2):219-233.

24. Harmon-Jones E. Contributions from research on anger and cognitive dissonance to understanding the motivational functions of asymmetrical frontal brain activity. Biol Psychol. 2004;67(1-2):51-76.

25. Sutton SK, Davidson RJ. Prefrontal brain asymmetry: a biological substrate of the behavioral approach and inhibition systems. Psychol Sci. 1997;8(3):204-210.

26. Davidson RJ. Brain asymmetry, the emotions, and mood disorders. Harv Ment Health Lett. 1992;9:4-5.

27. Wheeler RE, Davidson RJ, Tomarken AJ. Frontal brain asymmetry and emotional reactivity: a biological substrate of affective style. Psychophysiology. 1993;30(1):82-89.

28. Balconi M, Finocchiaro R, Canavesio Y. Reward-system effect (BAS rating), left hemispheric "unbalance" (alpha band oscillations) and decisional impairments in drug addiction. Addict Behav. 2014;39(6):1026-1032.

29. Buss KA, Schumacher JR, Dolski I, Kalin NH, Goldsmith HH, Davidson RJ. Right frontal brain activity, cortisol, and withdrawal behavior in 6-month-old infants. Behav Neurosci. 2003;117(1):11-20.

30. Sobotka SS, Davidson RJ, Senulis JA. Anterior brain electrical asymmetries in response to reward and punishment. Electroencephalogr Clin Neurophysiol. 1992;83(4):236-247.

31. Schutter DJ, de Haan EH, van Honk J. Anterior asymmetrical alpha activity predicts Iowa gambling performance: distinctly but reversed. Neuropsychologia. 2004;42(7):939-943.

32. Gianotti LR, Knoch D, Faber PL, et al. Tonic activity level in the right prefrontal cortex predicts individuals' risk taking. Psychol Sci. 2013; 20(1):33-38.

Neuropsychiatric Disease and Treatment

\section{Publish your work in this journal}

Neuropsychiatric Disease and Treatment is an international, peerreviewed journal of clinical therapeutics and pharmacology focusing on concise rapid reporting of clinical or pre-clinical studies on a range of neuropsychiatric and neurological disorders. This journal is indexed on PubMed Central, the 'PsycINFO' database and CAS,
33. Knyazev GG, Slobodskoy-Plusnin JY. Substance use underlying behavior: investigation of theta and high frequency oscillations in emotionally relevant situations. Clin EEG Neurosci. 2009;40(1):1-4.

34. First MB, Gibbon M, Spitzer RL, Williams JBW, Smith L. Structured Clinical Interview for DSM-IV ${ }^{\circledR}$ Axis II Personality Disorders (SCID-II), Interview and Questionnaire. Washington, DC: American Psychiatric Press, Inc.; 1997.

35. Beck AT, Steer RA, Brown G. Beck Depression Inventory-Second Edition (BDI-II). San Antonio, TX: Psychological Corporation; 1996.

36. Bechara A, Damasio AR, Damasio H, Anderson SW. Insensitivity to future consequences following damage to human prefrontal cortex. Cognition. 1994;50(1-3):7-15.

37. Bechara A, Damasio H, Damasio AR, Lee GP. Different contributions of the human amygdala and ventromedial prefrontal cortex to decisionmaking. J Neurosci. 1999;19(13):5473-5481.

38. Northoff G, Grimm S, Boeker H, et al. Affective judgment and beneficial decision making: ventromedial prefrontal activity correlates with performance in the Iowa Gambling Task. Hum Brain Mapp. 2006;27(7):572-587.

39. Klem GH, Lüders HO, Jasper HH, Elger C. The ten-twenty electrode system of the International Federation. The International Federation of Clinical Neurophysiology. Electroencephalogr Clin Neurophysiol Suppl. 1999;52:3-6.

40. Ludwig KA, Miriani RM, Langhals NB, Joseph MD, Anderson DJ, Kipke DR. Using a common average reference to improve cortical neuron recordings from microelectrode arrays. J Neurophysiol. 2009;101(3):1679-1689.

41. Semlitsch HV, Anderer P, Schuster P, Presslich O. A solution for reliable and valid reduction of ocular artifacts, applied to the P300 ERP. Psychophysiology. 1986;23(6):695-703.

42. Pascual-Marqui RD. Standardized low-resolution brain electromagnetic tomography (sLORETA): technical details. Methods Find Exp Clin Pharmacol. 2002;24 Suppl D:5-12.

43. Pascual-Marqui RD, Michel CM, Lehmann D. Low resolution electromagnetic tomography: a new method for localizing electrical activity in the brain. Int J Psychophysiol. 1994;18(1):49-65.

44. Palmero-Soler E, Dolan K, Hadamschek V, Tass PA. swLORETA: a novel approach to robust science localization and synchronization tomography. Phys Med Biol. 2007;52(7):1783-1800.

45. Knoch D, Pascual-Leone A, Meyer K, Treyer V, Fehr E. Diminishing reciprocal fairness by disrupting the right prefrontal cortex. Science. 2006;314(5800):829-832.

46. Bechara A, Damasio AR. The somatic marker hypothesis: a neural theory of economic decision. Games Econ Behav. 2005;52(2):336-372.

47. Boggio PS, Campanhã C, Valasek CA, Fecteau S, Pascual-Leone A, Fregni F. Modulation of decision-making in a gambling task in older adults with transcranial direct current stimulation. Eur J Neurosci. 2010;31(3):593-597.

48. Amiez C, Joseph JP, Procyk E. Reward encoding in the monkey anterior cingulate cortex. Cereb Cortex. 2006;16(7):1040-1055.

49. Davidson RJ. Affective style and affective disorders: perspectives from affective neuroscience. Cognition and Emotion. 1998;12(3):307-330.

50. Perry JL, Joseph JE, Jiang Y, et al. Prefrontal cortex and drug abuse vulnerability: translation to prevention and treatment interventions. Brain Res Rev. 2011;65(2):124-149.

and is the official journal of The International Neuropsychiatric Association (INA). The manuscript management system is completely online and includes a very quick and fair peer-review system, which is all easy to use. Visit http://www.dovepress.com/testimonials.php to read real quotes from published authors. 